\title{
Tomographic diffractive microscopy: towards high- resolution 3-D real-time data acquisition, image reconstruction and display of unlabeled samples
}

\author{
Jonathan Bailleul, Bertrand Simon, Matthieu Debailleul, Ludovic \\ Foucault, Nicolas Verrier, Olivier Haeberlé ${ }^{*}$ \\ Laboratoire Modélisation Intelligence Processus et Systèmes MIPS EA-2332, \\ Université de Haute-Alsace, \\ 61 rue Albert Camus 68093 Mulhouse Cedex, France \\ *Corresponding author: olivier.haeberle@uha.fr
}

\begin{abstract}
Tomographic diffractive microscopy allows for imaging unlabeled specimens, with a better resolution than conventional microscopes, giving access to the index of refraction distribution within the specimen, and possibly at high speed. Principles of image formation and reconstruction are presented, and progresses towards realtime, three-dimensional acquisition, image reconstruction and final display, are discussed.
\end{abstract}

\section{Introduction}

In recent years, microscopy techniques permitting to observe unlabeled samples, thus avoiding specimen preparation, have known a regain of interest [1-4]. For imaging samples not producing auto-fluorescence, possible microscopy techniques fall into two categories. Those based on non-linear optics [5], like second or third harmonic generation (SHG, THG), coherent anti-Stokes Raman scattering (CARS) or stimulated Raman scattering (SRS) have the advantage of structural or of chemical specificity, but, using femtosecond lasers, these techniques have the drawback of being more complex and expensive than linear imaging techniques. Furthermore, they deliver chemical information about certain molecules, but not global biophysical information for 3-D imaging, such as tissue/cell density refractive index, dry mass or birefringence properties.

On the contrary, techniques, which allow for recording both the amplitude and phase of the light diffracted by the specimen (by use of digital holographic recording [6], wavefront sensors [7], light-field cameras [8], via numerical reconstruction from the transport of intensity equation [9], or by DIC microscopy integration [10]), have the advantage of being more cost-saving and easier to implement. Digital Holographic Microscopy (DHM), or wavefront sensors for example are now established techniques, even commercially available [11]. The main drawback of DHM is its poor resolution along the optical axis. While the precision of measurement of interferometric approaches is unsurpassed [12,13], the resolution when reconstructing 3-D transparent specimens is plagued by the small quantity of information, which is recorded, because of the use of only a single illumination direction [14]. In order to increase the recorded quantity of information, a rotation of the

specimen [15-22], or a variation of the illumination wave inclination [23-28] can be used, and the set of recorded interferograms represents a diffractive tomographic acquisition [29,30]. This allows for a numerical 3-D reconstruction of the observed specimen. Recently, effective combination of sample rotation with illumination wave inclination tomography, as proposed in Ref. [31] has been performed [32], demonstrating for the first time isotropic- and high-resolution imaging in a complete Ewald sphere acquisition configuration. These various approaches are known under several names: tomographic phase microscopy, synthetic aperture microscopy, optical diffraction tomography, digital holographic microscopy, scanning holography microscopy, tomographic diffractive microscopy.

TDM being a numerically-based imaging technique, which computes reconstructed images from several interferograms, a real-time display of the observed sample images is difficult to achieve. This may trigger unease of use, for example when performing microscope adjustments (even for such simple tasks as sample 
positioning), or when observing moving, leaving samples. Two bottlenecks arise: first, one must acquire and transfer to the computer enough interferograms to perform the reconstruction, then, the image reconstruction itself creates a supplemental delay before displaying the image.

In this work, we first briefly recall the theoretical basis of TDM. Then, we present new developments and perspectives for image computation, which should allow for real-time reconstructions and display, which, combined with fast acquisitions, could allow for high-speed, high-resolution, 3-D imaging, opening new perspectives for experimentalists working on living samples, or studying dynamic phenomena.

\section{Basics of tomographic diffractive microscopy}

Tomographic diffractive microscopy (TDM) is a numerical imaging technique, which therefore relies on inversion methods to reconstruct an image of the observed sample. We here only give the basic equations to explain the main steps of the sample reconstruction [33]. On starts from the Helmholtz equation in an inhomogeneous medium:

$$
\left(\nabla^{2}+k(\vec{r})^{2}\right) \psi(\vec{r})=0 \quad \text { with } k(\vec{r})=2 \pi n(\vec{r}) / \lambda
$$

where $n$ is the complex refractive index of refraction and $\lambda$ is the wavelength in vacuum. Such an equation cannot be solved analytically in the general case, but can be simplified. The simplest approach applies for weakly diffractive specimens, considering that the first Born approximation is valid (which is often the case for transparent biological samples at the cellular level), so that the diffracted field amplitude is small compared to the incident field, so that the field inside the specimen can be approximated to the incident field. In that case, one shows that Eq. (1) can be rewritten as:

$$
\psi_{s}(\vec{r})=\int G\left(\vec{r}-\overrightarrow{r^{\prime}}\right) O\left(\overrightarrow{r^{\prime}}\right) \psi_{i}\left(\overrightarrow{r^{\prime}}\right) d \overrightarrow{r^{\prime}}
$$

with the Green function $G(\vec{r})=\exp \left(j k_{i}|\vec{r}|\right) /(4 \pi|\vec{r}|)$ and the object function $O(\vec{r})=k_{i}^{2}\left(n^{2}(\vec{r})-n_{0}^{2}\right) / n_{0}^{2}$. Rewriting Eq.(2) in Fourier space, leads then to:

$$
\widetilde{\psi_{s}}\left(\overrightarrow{k_{d}}\right)=\frac{1}{4 \pi k_{i}} \delta\left(\left|\vec{k}_{d}\right|-\left|\vec{k}_{i}\right|\right) \widetilde{O}\left(\vec{k}_{d}-\vec{k}_{i}\right)
$$

Equation (3) links the object function's Fourier transform to the Fourier transform of the complex amplitude of the scattered field. So, under Born assumption, the diffracted field simply depicts a subset of the 3-D Fourier transform of the observed specimen permittivity distribution The interested reader is referred for more details to classical textbooks on electromagnetism, for example Refs. [34,35]. More elaborated approaches have been developed (see e.g. Ref. [29] and references therein), when the sample under investigation is thicker, and/or presents stronger permittivity contrasts.

Taking into account the elasticity condition in linear optics on therefore has:

$$
\begin{aligned}
& \left|\vec{k}_{d}\right|=\left|\vec{k}_{i}\right| \\
& \vec{k}_{o}=\vec{k}_{d}-\vec{k}_{i}
\end{aligned}
$$

Equations $(4,5)$ now explain the construction of the object frequency support, therefore the entire image reconstruction procedure. So, in Fourier space (see Fig. 1), the diffracted wave is represented by a set of wavevectors, which depict the so-called Ewald sphere, from Eq. (4) (a circle in 2-D, for the sake of simplicity). Because of the microscope objective's limited numerical aperture, the actually recorded wavevectors belong to a cap of sphere only. Then, considering elastic scattering Eq. (5), these diffracted wavevectors are translated, in order to represent the object wavevectors (Fig. 1(a)).

In digital holographic microscopy, one uses a single illumination, and one can therefore record only very limited information about the specimen. In practice, one obtains a good lateral resolution, but the longitudinal resolution within the specimen is very poor [14]. Such a configuration in fact corresponds to a transmission microscope, which condenser numerical aperture would be null, while the resolution in transmission microscopy is indeed related to both the objective numerical aperture $\mathrm{NA}_{\mathrm{obj}}$ and the condenser numerical aperture NAcond [36]. 
In order to take benefit of the condenser numerical aperture to improve the lateral and longitudinal resolution, TDM in transmission also uses tilted illumination (Fig. 1(b)). In that case, and under the same elastic scattering approach, one records a shifted, complementary subset of information. For a large number of successive illuminations, a numerical synthetic aperture approach permits to accumulate information in the Fourier space, and an enlarged and filled frequency support is obtained (Fig. 1(c)). A final 3-D Fourier transform, from Eq. (3), permits to obtain the observed specimen reconstruction.

Figure 1(d) depicts a sketch and a picture of the actual setup we use, highlighting the specific elements to perform TDM. It is based on a classical Mach-Zehnder interferometer, with a coherence source $(633 \mathrm{~nm}$ HeNe laser) being split into a reference beam, and an object beam, with the specific addition that a fast tiptilt mirror (FSM 300 Newport) allows for precise controlling of the sample beam illumination angle, via a 60x $\mathrm{NA}=1.4$ objective (Zeiss).
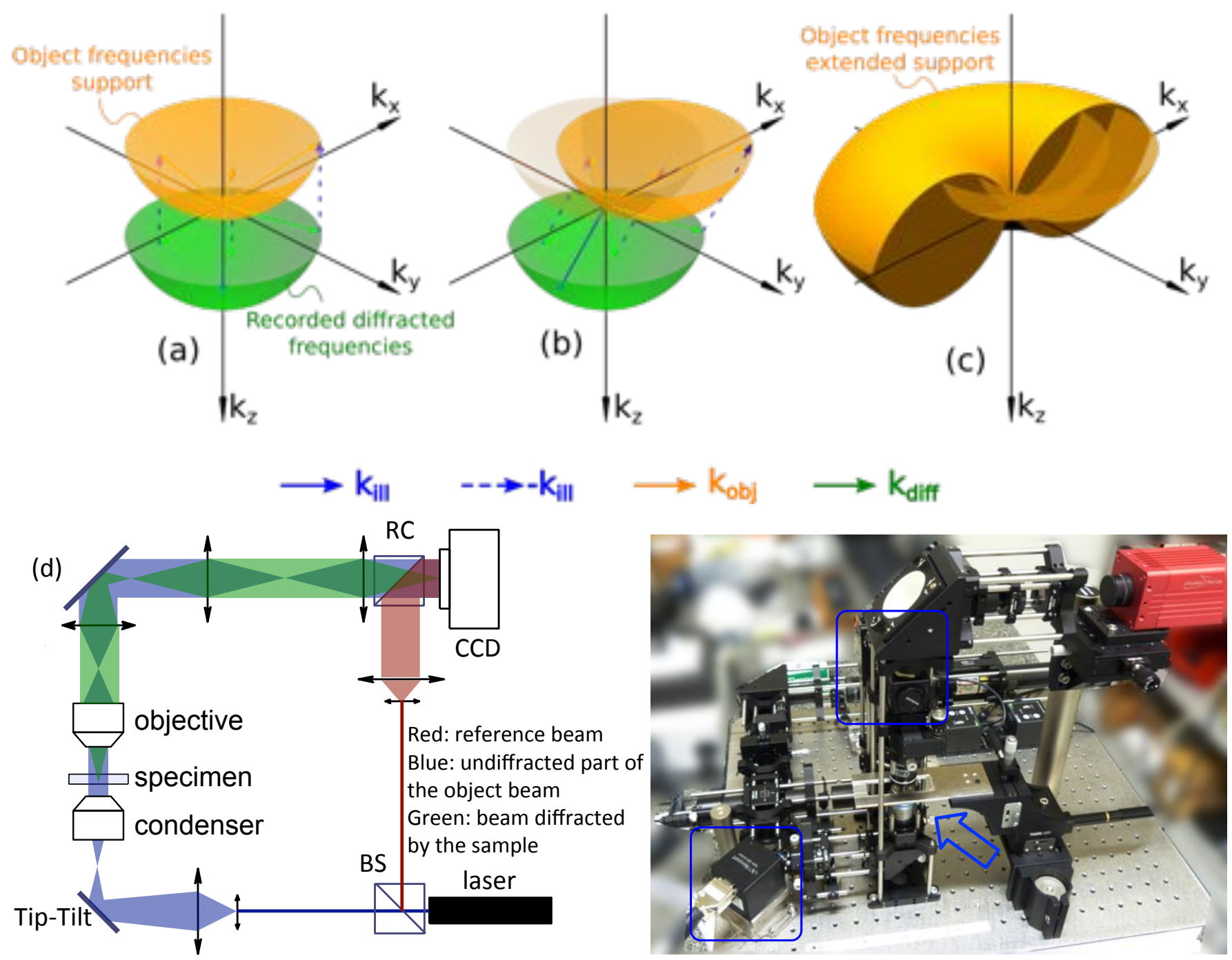

Fig. 1. Construction in Fourier space $\left(\mathrm{k}_{\mathrm{x}}, \mathrm{k}_{\mathrm{y}} \mathrm{k}_{\mathrm{z}}\right)$ of tomographic diffractive microscopy optical transfer function with illumination rotation. (a): normal illumination. (b): inclined illumination. (c): final OTF with many illuminations. Vector $k_{\text {ill }}$ represents the varying angle of incidence illumination wavector. According to the elastic scattering condition $\mathrm{k}_{\text {diff }}=\mathrm{k}_{\mathrm{obj}}+\mathrm{k}_{\mathrm{ill}}$, recorded diffracted wavevectors $\mathrm{k}_{\text {diff }}$ are back-translated to form the object wavevectors kobj support. (d): sketch of our present setup to perform TDM (BS: beam splitter RC: recombination cube) and picture of the system, highlighting the tip-tilt mirror and recombination cube (blue squares), and the two-objectives used as condenser and for detection (blue arrow).

Object beam is collected by a $100 \mathrm{x} \mathrm{NA}=1.4$ objective (Olympus), and is recombined with reference beam before the camera (Photonfocus MV1-D1312(I/IE)-100-GB), visible on the upper-right corner of Fig. 1(d)), in 
off-axis configuration (camera is running at 85 frames per second). Alternately, phase-shifting acquisition is also possible with an electro-optical modulator (Newport 4002, not represented here). The system is built using from-the-shelf prototyping opto-mechanical elements (Thorlabs), and installed onto an anti-vibration benchtop (Newport M-BT-2024-OPT02). A remotely controlled (Thorlabs MT1-Z8) x-y sample holder allows for fine adjusting of the sample, z-focusing being performed manually. The use of two high-NA objectives, as condenser, and for detection, implies small working distances, so samples are prepared between two $170 \mu \mathrm{m}^{-}$ thick cover glass. A special sample stage has been designed to handle such fragile preparations (appears in grey between both objectives on picture $1(\mathrm{~d})$ ). The camera finally records three contributions, the reference beam, for holographic acquisitions, the beam diffracted by the sample, which carries information about it (the cap of sphere Eq. (4)), and the non-diffracted part of the illumination beam, which allows for precise repositioning of the sphere cap in Fourier space (Eq. 5) [14].

The advantages of TDM, compared to classical transmission microscopy, are the following.

- A true 3-D imaging is possible for transparent samples, contrary to phase microscopy, which delivers surface reconstruction only, or integrated profiles.

- A better resolution is obtained, thanks to the use of coherent imaging, which permits to acquire specimen information, without the high frequency attenuation characteristic of the use of incoherent light. Note that the frequency support has the same shape than for a conventional transmission microscope, but thanks to the better frequency transmission, the lateral resolution can theoretically be doubled [25], in transmission [37,38], as well as in reflection [39,40]. In transmission, a resolution of $130 \mathrm{~nm}$ using a HeNe laser, or $\lambda / 3.5 \mathrm{NA}$ has been obtained [37]. More recently, using a $405 \mathrm{~nm}$ source, Cotte et al. demonstrated a $90 \mathrm{~nm}$ resolution [41] (or $\lambda / 3.2 \mathrm{NA}$ ), compared to $\lambda 2 \mathrm{NA}$ in incoherent microscopy (and considering that $\mathrm{NA}_{\mathrm{obj}}=\mathrm{NA}_{\text {cond }}$ ).

- TDM and its variants give access to the index of refraction distribution within the observed specimen, at the microscopic scale, and the interest of this non-labeling imaging modality has been widely demonstrated for biological investigations as well as for studying artificial structures [15-28,32,37,38,4145]. Note that the index of refraction is a complex quantity, related to the refraction and to the absorption. This dual imaging capability has not yet found applications for biological studies, but could used to distinguish organelles from their refraction/absorption properties [14,25,26,32,37,38,46,47], and not from their refraction only, as usually performed.

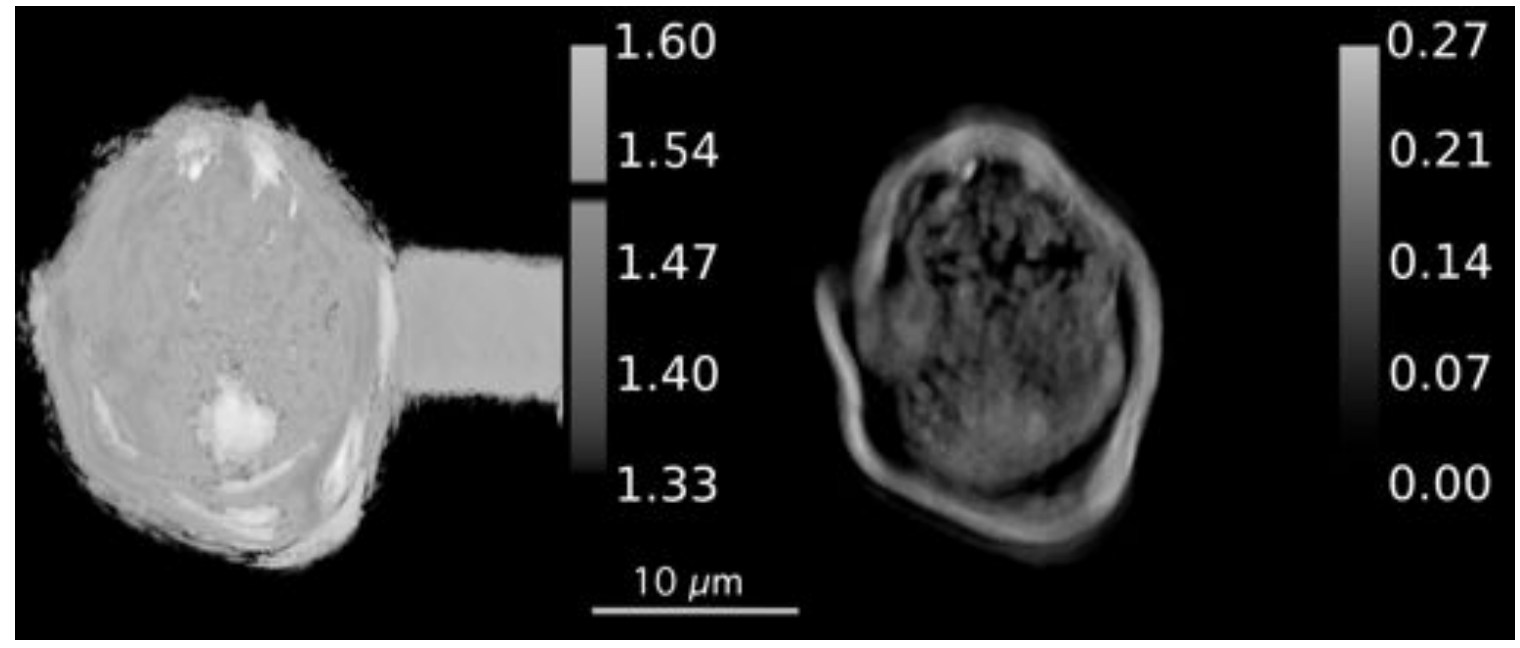

Fig. 2. Betula pendula pollen grain observed with TDM. Left: refraction image. Right: absorption image.

Figure 2 shows a Betula pendula pollen grain, attached to a photopolymer tip for manipulation, displaying refraction and absorption images. Note the very different aspect of the pollen on both images, and the photopolymer tip, visible on the refraction image, but not on the absorption one (see Ref. [32] for acquisition details). These images highlight the inner structure of the pollen grain. The knowledge of the photopolymer tip and immersion medium indices of refraction allows for calibration. The pollen walls appear composed of high refraction index components. The inner part is globally made out of lower refractive index components. 
The absorption image shows that the absorptive components are mostly concentrated in the pollen walls, which appear as a bright structure surrounding the pollen nucleus (which is also slightly absorptive, but separated from the pollen walls by a non-absorptive layer ((Fig. 2, right).

Other approaches to fill the Fourier space exist, by rotating the specimen [15-22], changing the illumination wavelength [48,49], recording the reflected wave [39,40], or combining transmission and reflection [25]. Rotating the specimen requires a large number of sample rotations (typically one per degree [19,50]), which may be difficult to perform at microscopic scale. Furthermore, the ultimate possible resolution is lower than with illumination rotation [29], and Fourier space filling is not complete, leaving missing frequencies along the rotation axis [51,52]. Changing the illumination wavelength has also proven to be a successful approach [48,49], and the development of white-light illumination approaches [53,54] has suppressed the need for wide spectrum coherent sources, but anisotropic resolution is obtained, because of the peculiar shape of the Optical Transfer Function (OTF) in this configuration [29,43].

However, tomographic diffractive microscopy up-to-now suffers from a major limitation. In terms of information processing, incoherent microscopes (transmission, phase contrast, DIC...) make the best use of their condenser, and are parallel systems of information processing, as the specimen is illuminated over many incidences simultaneously. The price to be paid is a lower resolution and a loss of information (intensity-only images are obtained), but very high-speed imaging is possible, limited in practice only by the acquisition camera performances. For tomographic diffractive microscopy, the data acquisition process must be sequential, one illumination angle at a time, which limits the speed, a possible drawback for studying living specimens of fast phenomena, and the necessary computer-based image reconstruction further delays the display.

In the next sections, we present a GPU (Graphics Processing Unit)-based image reconstruction method, which can deliver several 3-D reconstructions per second, so may help for a wider adoption of this new microscopy technique.

\section{Real-time image reconstruction strategy}

Tomographic diffractive microscopy in transmission is usually performed by first acquiring numerous interferograms (n: typically a few tens to a few hundreds), then by computing and displaying the image reconstruction of the sample. For typical configurations of our setup (output: real and imaginary volumes of $512^{3}$ voxels in single-float precision, input: $\mathrm{n} \times 1024^{2} 8$ bits interferograms with off-axis acquisition and $4 \mathrm{n} \times 512^{2}$ with phase shifting acquisition), we already managed to reduce acquisition and reconstruction time below one minute each $[26,34,55]$. Provided one can accelerate and parallelize both processes, real-time acquisitions and reconstructions could be achieved. This would allow for user supervision of specimens during acquisition, which, for example, could be very relevant for routine acquisitions.

A high-speed tomographic configuration has been proposed in Ref. [56], data are acquired and stored by a fast camera, which allows for imaging a live sample at several 3-D frames per second. Though, only off-line reconstruction is possible, due to insufficient transfer rates to a computer, and reconstruction speed. Indeed, on-line 4-D visualization is very challenging due to the amount of data to be transferred from camera to computer, and to be processed for real-time reconstruction. A scheme optimizing the reconstruction sequence in order to match high acquisition speed would allow for live imaging and display.

The method presented in [56] was amended in [57] in order to achieve such a live imaging, but due to the limited camera transfer rate, the size of both 2-D interferograms and 3-D reconstructions is drastically reduced. A fast live 3-D reconstruction method was presented in [58], which also demonstrates that increasing the size of interferograms and reconstructions volumes decreases the reconstruction speed due to the volume of data to process. Though, the tomographic setup providing input interferograms does not match the speed of the setups shown in [56,57], so the live reconstruction is actually simulated from recorded data. In this paper, we use the TDM setup presented in [32] (without the specimen rotation stage) to perform fast and real-time imaging of a static specimen with maximum size of interferograms and reconstruction, which should extend to real-time imaging of live specimen via scaling both acquisition and reconstruction hardware and possibly decreasing the size of the reconstructed volume. 


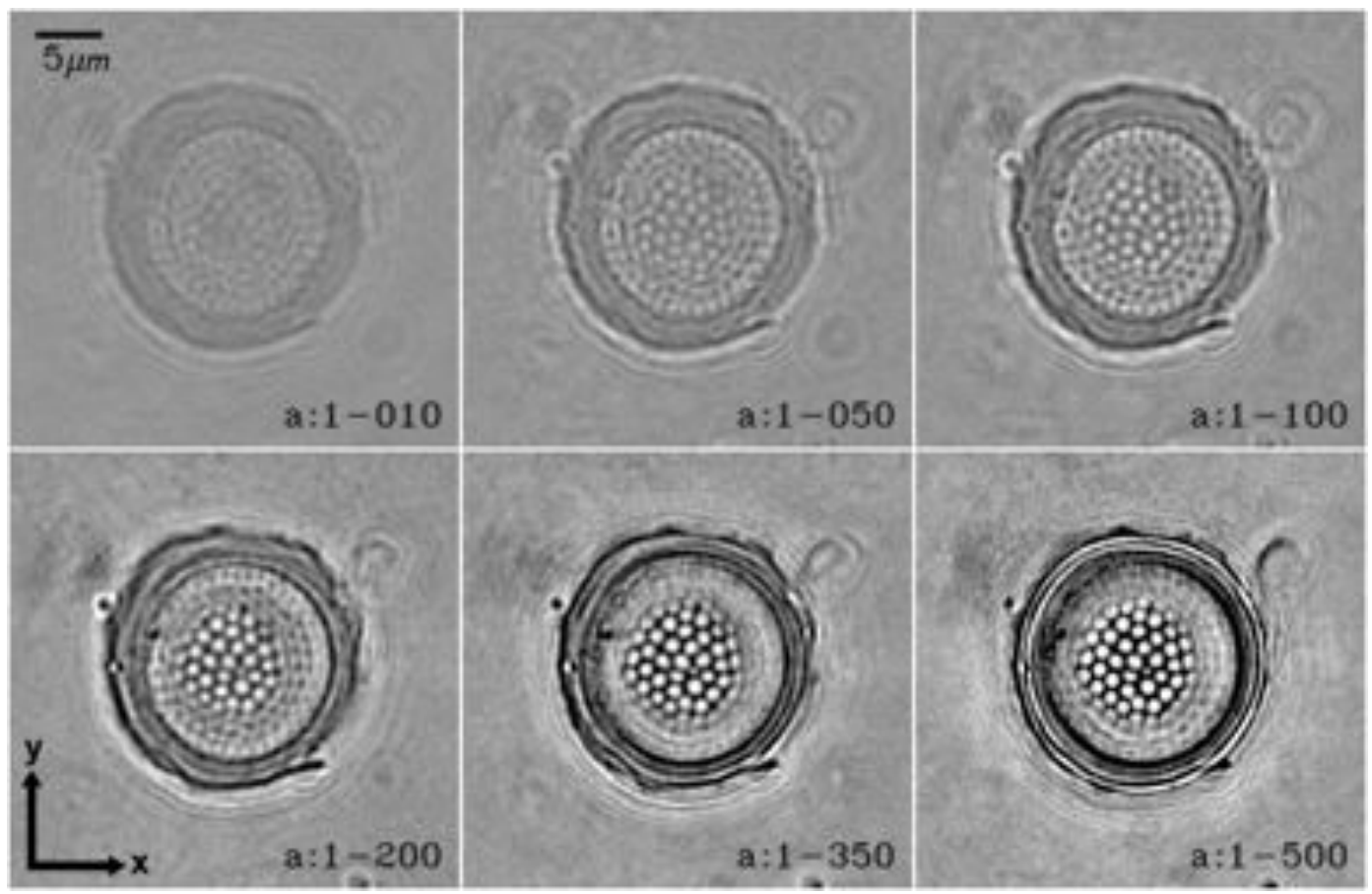

Fig. 3. Slice ( $\mathrm{z}=240 / 512)$ extracted from successive $512^{3} 3-\mathrm{D}$ tomographic reconstructions of a diatom shell with increasing number of illuminations, synthetizing an increasing numerical aperture at illumination.

Note the progressive apparition of smaller details (See also Media 1).

For a CPU (Central Processing Unit) setup, the high-performance computing techniques we have considered [59-61] propose acceleration factors ranging from 10 to 100 depending on the hardware architecture and the algorithm profile. In our case, this would allow for computing several full 3-D image reconstructions during the acquisition sequence, which represents a view with increasing level of details of the specimen. This, for example, would allow for detecting positioning mistakes or other flaws in sample preparation. As detailed in the following, computation is indeed incremental, due to the accumulation of sphere caps during angular scanning [14]. As a consequence, it becomes possible to compute 3-D reconstructions of increasing level of detail at regular time intervals, while increasing the number of angles used for the reconstruction (so the synthetic numerical aperture at illumination) as seen in Fig. 3 (see also Media 1). Dynamic synthesis of an increasing numerical aperture condenser at illumination would for example allow the user for stopping the acquisition upon a visual observation with satisfactory reconstruction level of details instead of running a static scheme until completion, or using a priori guess.

We first present a hardware-software system implementing progressive image reconstruction, which will allow for identifying bottlenecks of the reconstruction process. We then present CPU code acceleration, able to deliver a 3-D image every second on a high-end Desktop PC. Changing the hardware architecture to GPU, we finally present a hybrid GPU/CPU scheme able to provide several 3-D frames per second thanks to an additional scientific computation board.

\subsection{CPU-based progressive reconstruction computing}

The image reconstruction procedure is depicted in Fig. 4, which described the implementation of the process described in Section 2. A hologram acquisition is performed for each illumination angle (with phase shifting holography, 4 interferograms are acquired in order to compute one hologram, off-axis holography can also be used). Each hologram is 2-D Fourier transformed, then projected onto a cap of sphere, which position in Fourier space is given by the current illumination angle (cf Fig. 1 (c)). Sphere caps are accumulated in a 3-D Fourier volume. As they often overlap, normalization is applied to preserve consistency of frequency values. 
Mapping of the 2-D holograms onto the 3-D cap of sphere is an important step not to overlook, because it implies projecting a rectilinear grid (2-D holograms) onto a discretized cap of sphere embedded in a $3-\mathrm{D}$ volume used for Fourier transforms. For coarse discretization, this may imply phase and amplitude errors when projecting the Fourier coefficients. For our experiments, we use a basic projection, with simple interpolation of the data. This procedure proved to be sufficient in practice [14,26,32,37-40], as we use fine discretization of the field of view, and because fast illumination angular scanning is made, which permits to record many holograms. It has to fortunate consequences: averaging the inevitable noise, because we have data redundancy, and also averaging the residual interpolation errors. For speeding acquisition, one could use a lower number of illuminations, but more accurate reconstruction procedure would have then to be implemented.

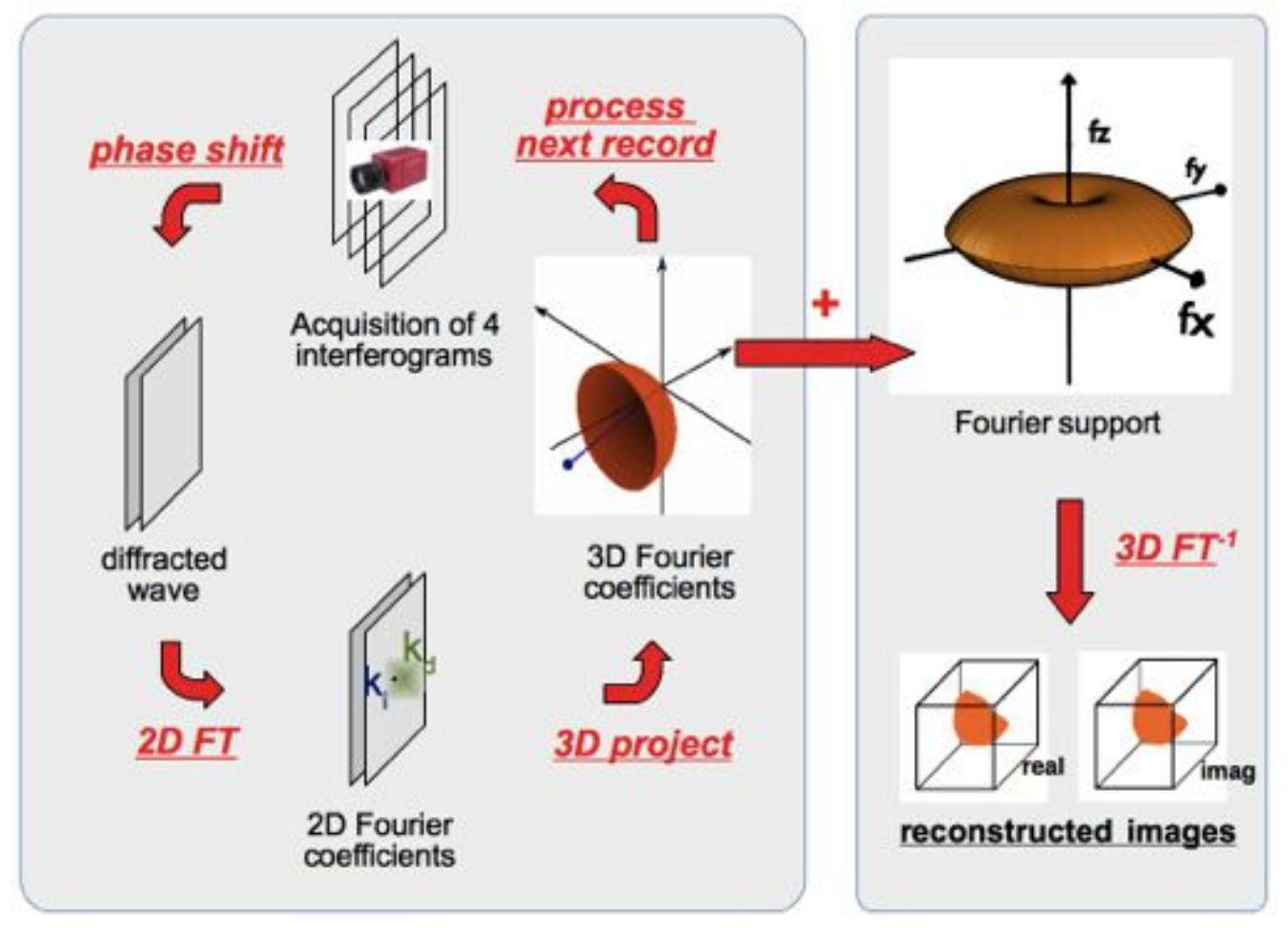

Fig. 4. Outline of 3-D image reconstruction in TDM. Left: computing of sphere caps of object frequencies from angular scanning holographic acquisition, 2-D FFTs and projections on cap of spheres. Right: 3-D image reconstruction update via a 3-D inverse FFT after normalization of accumulated coefficients.

An inverse 3-D FFT then raises a complex 3-D image of the specimen, the level of detail depending on the number and spreading of accumulated caps (Fig. 3). It can then be displayed to the user, showing either or both real and imaginary parts on an interactive 3-D slicer displaying $2 \mathrm{D}$ cuts in xy/xz/yz planes.

We have implemented this reconstruction procedure on the same middle-range PC, which performs acquisition, and features a Core i7 860 (quad-core CPU) with 16GB of RAM (DDR3 $1600 \mathrm{MHz}$ in dualchannel). The PC is linked to the camera using a GigE interface for conveying snapshot orders and resulting images. All other devices (i.e. rotating mirror and phase modulator) are piloted by a DAC (Digital Analog Converter) system linked by USB and work fast enough to sustain acquisition at maximum camera rate for our interferogram sizes [55].

The choice of programming language for such a heterogeneous system is of importance. The pros and cons of different programming language in the various domains of instrumentation, scientific computing, data management are very diverse, and often contradictory, as some languages are better suited for easy programming of tasks like data acquisition from external devices (such as cameras), while others are more efficient in terms of pure computation power. Because we intend developing an integrated tool for data acquisition and management, as well as image reconstruction and display, and having to handle 
input/output buses, CPU and GPU computation, as well as final display, we choose to develop the system in $\mathrm{C} / \mathrm{C}++$ language. Our choice is motivated by the fact that $\mathrm{C} / \mathrm{C}++$ is a very universal language in this case, and because manufacturers of the various subsystems we use (camera, tip-tilt mirror command, as well as GPU card) all offer $\mathrm{C} / \mathrm{C}++$ routines and/or precompiled drivers, which are then easier to assemble with our own code.

The software architecture for the progressive reconstruction program is the following. A single program controls the parallel execution of 3 threads (sub-processes), which collaborate in using common data in memory banks shared by all threads (See Fig. 5).

- The acquisition process produces interferograms as fast as possible, stacking these in shared memory.

- The reconstruction process starts by emptying the entire stack, then computes the corresponding new caps, and finally computes an updated 3-D reconstruction of the specimen, which is placed in shared memory. Then it goes on with the new interferograms, which were stacked during the former reconstruction update, until acquisition stops.

- The visualization process executes a 3-D slicer displaying the most recent $3-\mathrm{D}$ reconstruction and listening for user interaction orders (slice navigation, zoom, etc).

Such a 3-threaded program runs very smoothly on a quad-core CPU: visualization and acquisition are not intensive processes unlike the reconstruction one, which stresses only one CPU core under a standard programming architecture. This scheme automatically adapts to various computing capacities and acquisition speeds and provides the best 3-D reconstruction rate available in this case. With our reconstruction method, we may reduce the output volume size (zoom out) if we need a reconstruction speed boost (e.g $256^{3}$ is 8 times smaller than $512^{3}$ ).

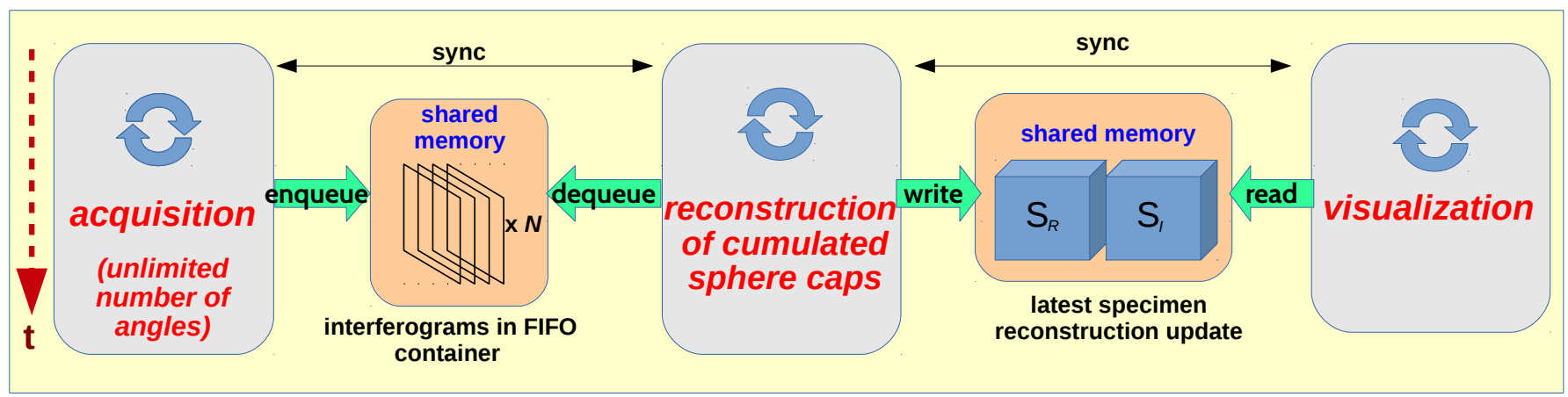

Fig. 5. Principle of the three-thread program for parallel execution of acquisition, reconstruction, and display of TDM images.

With a straightforward implementation of CPU code [62], computing and accumulating a projected sphere cap takes $25 \mathrm{~ms}$ (Fig 4, left). Computing a reconstruction update from an additive cap accumulation takes 3.5 seconds (Fig. 4 right), including $800 \mathrm{~ms}$ for FFT 3-D alone. It is clear that the latter part represents the bottleneck of our program. Since it requires in-depth optimization, we will first detail the computation steps it contains.

While Figure 5 describes the reconstruction process in terms of computing tasks to be processed in parallel, Fig. 6 describes it in terms of memory volumes to be used. Volume A stands for the 3-D Fourier volume where successively acquired sphere caps are accumulated (see Figs. 4 and 5). When angularly scanning the illumination, successively acquired cap of spheres intersect (see Fig. 1), meaning that some pixels in Fourier space are acquired several time. Volume $\mathrm{O}$ counts voxel-wise overlaps between caps, used to average the corresponding measurements, which is very favorable in terms of improving final reconstructions signal-tonoise [37]. After each new acquisition, a new normalized volume $\bar{A}$ is computed. Then, this volume must be converted to a memory organization $S_{\bar{C}}{ }^{1}$ compatible with the FFTW ("Fastest Fourier Transform in the West") algorithm [63], here chosen for its speed and efficiency. After FFTW Fourier transform is performed, the obtained image $S_{C}$ must again be converted into a volume $S$ for standard image display. 


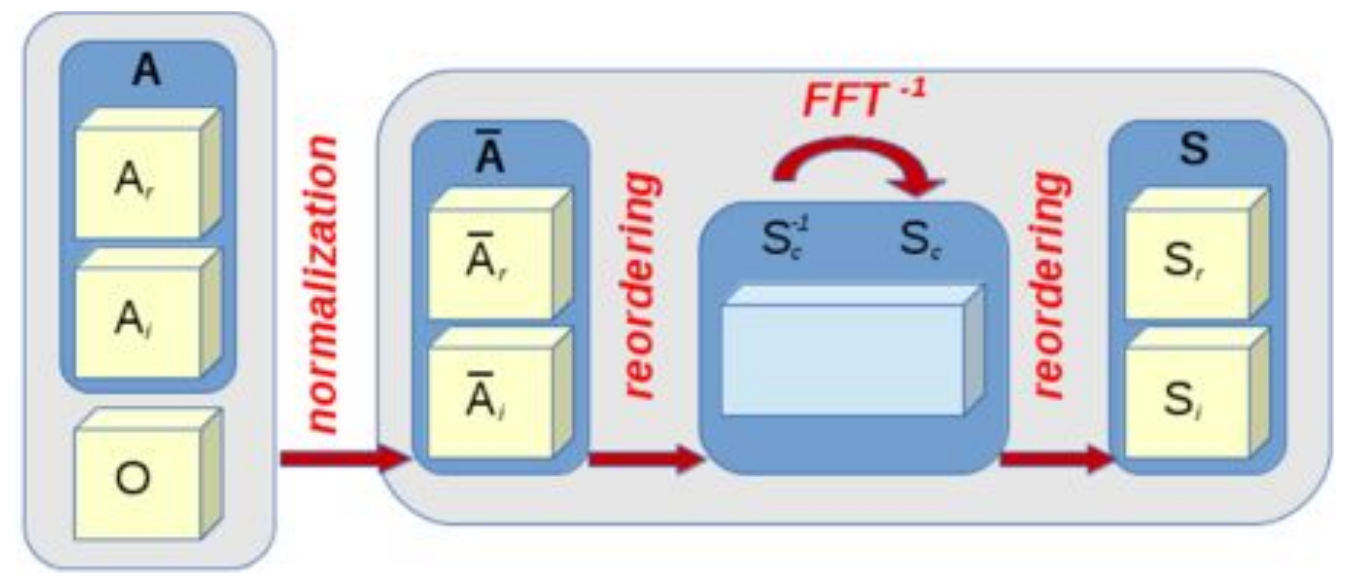

Fig. 6. Schematic for updating an image reconstruction in TDM (volume S, right) from the current state of a cap sphere accumulator volume (A left), which has to be normalized, reordered for efficient Fourier transform, the result of which being again reordered for display.

Indeed, FFTW implements a $3-\mathrm{D}$ Fourier volume with one array of complex values $\left(\mathrm{r}_{1}, \mathrm{i}_{1}, \ldots, \mathrm{r}_{\mathrm{n}}, \mathrm{i}_{\mathrm{n}}\right)$, which is known to allow for faster arithmetics via vectorization [64,65]. On the contrary, visualization of either real or imaginary part requires consecutive scalar values $\left(r_{1}, \ldots, r_{n}\right)$ and $\left(i_{1}, \ldots, i_{n}\right)$ in video memory. Thus one needs to tangle, and then detangle components, so as to switch between both representations. Furthermore, because of FFTW frequency ordering conventions [65], one has to perform a circular permutation of data (8 sub-cubes in 3-D, or 4 sub-squares in 2-D) in order to be able displaying a new image after Fourier transformation. In the following, we will name data reordering the succession of tanglings/detanglings/permutations, required to switch from memory organization required by FFTW to memory organizations required for display.

One can note that using the correct memory scheme, as required by FFTW, for every computations before the Fourier transform is performed, would spare data movements. However, doing so has impact onto performances of these preliminary computations: we measured on further optimization steps that it would in fact increase overall computation time [55].

We timed the computation units involved in this update. Concerning the parts we coded in C++, normalization takes $320 \mathrm{~ms}$, and each reordering takes $1.1 \mathrm{~s}[62,66]$ with maximum automatic optimizations inferred by the compiler (gcc 4.7).

The FFT part depends on the FFTW library: while default settings take $5 \mathrm{~s}$ of computation, activating multithreading and other FFTW specific optimizations (i.e pre-computed "wisdom" parameter files [65]) permits to decrease this time to $850 \mathrm{~ms}$. Such acceleration is drastic in comparison with the default setting, and it eventually turns out to be faster than most of our own code. Indeed, FFTW (such as Intel MKL (Math Kernel Library)) is reputed for the excellent level of algorithmic and source code optimization [65], on top of which compiler and architecture optimizations apply too.

Upon this observation, we worked to produce optimized code that could benefit from such a drastic acceleration on the present CPU setting.

An important point to consider in the reconstruction procedures is the possible phase unwrapping procedure, which has to be implemented for larger samples, or when the index of refraction contrast between the sample and its preparation medium is important. The phase unwrapping procedure is unnecessary when imaging very small samples, or when the index of refraction of the preparation medium is carefully chosen, which we pay attention to when preparing our samples. For samples requiring phase unwrapping, we implemented and compared two methods [67,68]. Processing $256^{2}$ pixels, 64 bits encoded holograms, requires about $20 \mathrm{~ms}$ with a FFT-only based algorithm [67] and $35 \mathrm{~ms}$ when using an algorithm based on a reliability function and a non continuous path [68]. At this time, such performances would slow down the data acquisition process (85 fps or 12ms). A second point is time consumption of data storage. At present time, images are recorded at full speed camera on a Solid State Disk (SSD), and in RamDisk for processing, so this process does not slow down the reconstruction pipeline. This is because we use a relatively slow camera. For high-speed cameras, more modern transfer protocols and buses should be used (see 3.3). 


\subsection{Accelerating CPU reconstruction}

A wide array of CPU optimization techniques described in [53,60] has been tested on division and reordering codes, but did not prove efficient. On the contrary, thread parallelization and code vectorization proved very efficient in the FFTW implementation [63,65].

Parallel tasks allows for addressing all available CPU cores for intensive computations. All computation steps involved in reconstruction are performed in sequential order; though, within each step, computations can be performed in parallel since these consist in sequential iterations over long arrays of independent elements (streaming computations [61]). OpenMP (Open Multi-Processing) parallel-for directives [71] were used and managed to both share computations between threads and to improve the global memory bandwidth [61].

Vectorization relies on SSE (Streaming SIMD (Single Instruction, Multiple Data) Extensions) (AVX: Advanced Vector Extensions) instructions and registers present in all modern CPUs [64,69]. Vectorization is a single-instruction on multiple data (SIMD) execution scheme, which relies on data-oriented parallelism to compute some sequences of arithmetic operations, typically 4 times faster. Furthermore, SSE also introduce specific streaming-oriented instructions, which can bypass the CPU cache mechanism and address main memory directly, and doubles the writing speed in that case. Though, in order to benefit from such speedups, the inner loop code snippets must be rewritten entirely in SIMD paradigm, using for instance SSE/AVX compiler intrinsic instructions [64]. Such rewriting is well covered by specific High Performance Computing (HPC) literature, but is not trivial [64,69].

In applying successive layers of code optimization like parallelization, vectorization, streaming, plus a set of algorithmic optimizations derived from experimentation [55], we eventually reduced the 3-D reconstruction update time from to $850 \mathrm{~ms}$ per 3-D update in executing our program on a Core i7 3820K, quad-channel DDR3 $1800 \mathrm{~Hz}$ CPU [66].

This CPU computation time is very competitive and supersedes the times reported for the CPU versions of the programs described in [57,58]. Comparing with [52], running with 73 interferograms of same size $\left(1024^{2}\right.$ 8 bits), our program computes equally fast. Though, it provides a $512^{3}$ output volume, which is 512 times bigger than the $64^{3}$ one in [58], and the 4 -core $3820 \mathrm{~K}$ CPU used in this work is at least 2 times less efficient than the 8-core Core i7 5960X CPU used in [52]. This CPU program also runs faster than the GPU version presented in [57] for $256^{3}$ outputs.

Our measurements show that memory bandwidth is the limiting factor: we managed to achieve an actual $18 \mathrm{~GB} / \mathrm{s}$ bandwidth [55] (21 GB/s theoretical limit of our PC setup). We also tested a more powerful PC setup featuring 2 CPUs, each one equipped with dedicated memory, thus doubling the overall memory bandwidth. Even in this case, the 3-D FFT test still takes $375 \mathrm{~ms}$ on a high-end PC (9 threads run on 2 hexa-core Xeon 2630, each with quad-channel $1333 \mathrm{MHz}$ DDR3 memory). Indeed it proves very difficult and expensive to scale further in speed using a CPU-only architecture [49].

A $1 \mathrm{~Hz}$ reconstruction rate is satisfactory for monitoring acquisitions, or adjusting the setup. Though, it is still too slow for 3-D video applications undertook in $[57,58]$. We therefore also considered GPU optimization.

\subsection{Real-time reconstruction on GPU}

GPUs have been used for general-purpose programming for the last 10 years, becoming a standard in HPC $[55,64]$ (alternatives like Intel MIC [70] now seem equally efficient). In the DHM field, GPUs have already been considered [56-58,62,66]. Since NVidia granted us a computing GPU suitable for our complete computation, we managed to improve on the seminal works presented in $[62,66]$.

The NVidia Tesla C2075 (Fermi series) is historically the first scientific-class computing GPUs proposing enough on-board memory ( $6 \mathrm{~GB}$ ) to fit all the data required for our progressive image reconstruction scheme (Fig. 8). With this card, the cuFFT library only takes $95 \mathrm{~ms}$ for computing our 3-D FFT, mostly thanks to the $144 \mathrm{~GB} / \mathrm{s}$ peak bandwidth compared to the $21 \mathrm{~GB} / \mathrm{s}$ on our CPU system. Though, we need to convert all our CPU C++ code to CUDA GPU code.

CPUs are perfectly suited for sequential programs with a high arithmetic to memory ratio [61]. Conversely, GPUs have been designed for fast data-access and the growing general-purpose use of the hundreds of dedicated shader units progressively made implementation of standard computations possible [64]. 
Memory accesses are a central part of the CUDA (Compute Unified Device Architecture) system. Focusing on hardware, CPUs use DDR3 memory, which balances bandwidth and latency, while GPUs use GDDR5 (Graphics Double Data Rate, version 5) memory with high bandwidth.

In CUDA, the programmer defines a computing grid covering all the data arrays to process, and the code to be executed by a single thread on a single array element at once and of unknown position in the array (a.k.a shader code). The CUDA subsystem instanciates all threads at once, and selects those for execution when their data is ready, allowing for maximum effective bandwidth, and making perfect use of the massive computation parallelism provided by the hundreds of shaders [72]. But this comes with huge restrictions in the GPU code, which must be largely rewritten [72], with GPU SIMD in mind.

Another constraint comes from the fact that GPU stands in an additional card. The PCIe bus it occupies has not been designed for data bandwidth, its 2.0 version barely reaching $6 \mathrm{~Gb} / \mathrm{s}$ when a standard CPU accesses main memory at $21 \mathrm{~GB} / \mathrm{s}$. This makes copies of volumes very slow [62]. As a consequence, it was mandatory to migrate all computations to GPU: in input, we then only copy interferograms (1 Mb per angle). No output copy of the reconstructed volume is required since the Tesla C2075 is both a computing- and a video card. Eventually, our CPU-to-GPU reconstruction code manages to compute a reconstruction update in only $230 \mathrm{~ms}$ [66], which allows for real-time 3-D update on the slower versions of our TDM setup [40,62] and still provides fast-paced 3-D monitoring of our latest TDM setup acquiring at 85 angles per second [32].

We then addressed the case of live 3-D use using our setup, which only supports a 85 fps $1024^{2}$ interferograms stream. Provided we can copy such data to GPU and compute a whole new reconstruction in $1 \mathrm{~s}$ at most, we would achieve a decent 4-D visualization with $1 \mathrm{~Hz}$ temporal resolution, and $1 \mathrm{~s}$ of delay regarding the current state of the specimen. With a unit projection time of $3 \mathrm{~ms}$ per interferogram [49], a 85block copy time of $30 \mathrm{~ms}$ and a 3-D unit reconstruction time of $230 \mathrm{~ms}$, we can reconstruct 85 interferograms in $550 \mathrm{~ms}$ only, idling $450 \mathrm{~ms}$ until the interferograms for the next 3-D frames start being recorded.

But with our setup, we usually need from 200 to 300 interferograms so as to obtain a full-resolution 3-D scene: so the bottleneck is now set on angular scanning and camera transfer to the PC memory.

In [56], the authors demonstrated acquisition speeds of $1000 \mathrm{fps}\left(1024^{2}\right.$, off-axis $)$ for off-line imaging. But for live imaging in [57], the $100 \mathrm{MB}$ transfer rate of their camera forces to drastically reduce the set of interferograms, so to use iterative 3-D interpolations to extrapolate missing data using a very timeconsuming method. The method detailed in [58] is way more efficient, but scanning or transfer rates are not mentioned since the live reconstruction is simulated from recorded data.

We suggest to overcome the camera-to-PC bottleneck in using recent cameras, allowing for a continuous stream of $600 \mathrm{fps}$ at $1024^{2} 8$ bits [55]. With such configuration, one could address $2 \mathrm{~Hz}$ (300 interferograms per 3-D frame) or $3 \mathrm{~Hz}$ (200) live reconstruction rates for 2 volumes of $512^{3}$ voxels at full resolution, which would be, to the best of our knowledge, unmatched so far. Our projections [49] show that our current GPU reconstruction method will follow this pace, using overlapping of 3-D loops already developed for the CPU version, CUDA-streaming for interleaving the copy and processing of interferograms like detailed in [72] and successfully applied in [58], and use of a Kepler [58] or Pascal Tesla GPU instead of the Fermi generation dating from 2011. We are now working towards such a $600 \mathrm{fps}$ simulation. We have also adapted for transmission tomography the method of aberration compensation we developed for tomography in reflection [40]. Their GPU implementation is expected to be very efficient and could be used to fill the idle GPU time while waiting for acquisition of the next 3-D frame.

Note that GPU processing has also proven to be very effective for fast implementation of the phase unwrapping preprocessing of the holograms. In Ref. [73], Backoach et al. have demonstrated processing rate of almost 2000 frames per second for $256^{2}$ holograms, showing that real-time phase unwrapping is possible even for high-speed cameras.

\subsection{Real time 3-D display}

At this point, we have a quasi real-time reconstruction system, capable of generating to-be-displayed 3-D data at a more than 3 images/s rate. Most of time, these data are then post-processed to produce visualizations on $2-\mathrm{D}$ displays that are easily interpretable by the user. Then, conventional softwares, such 
as those usually employed for 3-D image synthesis, can be used (see, for example, the methods proposed in [74] and the volumetric renderings in [32]).

One has however to realize that the whole chain that we use for acquiring data is indeed symmetrical to $3-\mathrm{D}$ holographic projection display devices, where a 3-D synthesis image serves as a basis for generating holograms $(\mathrm{CGH})$, which are then projected thanks to various devices (DMD, SLM, AOM), in order to achieve a three-dimensional rendering. For example, systems presented in [75] highlight the similarities between holographic display systems and TDM, in particular in regards to the key points of these techniques:

- The ability to perform fast calculations on holographic data [62,76-78].

- The ability to sequentially address display devices (or acquisition devices for TDM) at high refreshing rates $[62,60]$.

Moreover, the use of 3-D holographic projection in surgical applications [79,80], has shown that this type of interface allows for a more precise volumetric data understanding than the conventional displays on $2-\mathrm{D}$ screens. Finally, augmented reality microscopy systems have been reported, allowing for combining the views coming from microscope with additional information [81].

Consequently, TDM could evolve through a combination of these different approaches.

- First, the ability of TDM to directly produce holographic data could permit to relieve the onerous process of hologram computation from synthetic objects and computation would be limited to the techniques that we have already implemented [32,40,62] They just would have to be adapted to specificities of hologram projection.

- Secondly, holographic projection permits to relax the time-consuming 3-D data reconstruction task and their post- processing allowing for volumetric rendering.

- Finally, holographic displays that implement reduced viewing window thanks to eye tracking drastically reduces the holographic projections to be computed, therefore the number of angles to acquire in TDM process implementing a holographic display.

As a consequence, the released computation power could be reallocated to real-time computation of holographic projections containing additional information. So, co-conception approaches, taking into account both TDM and holographic display, may allow for a real-time augmented holographic display for TDM.

\section{Conclusion and perspectives}

Developments in tomographic diffractive microscopy by several groups in the world attract the interest of more and more end-users, thanks to the improved resolution, and/or very high precision measurements, and/or possibility to work on unlabeled samples.

Among present limitations of the method are its sequential nature, which limits speed acquisition, and its computational nature, which limits image delivery.

A high-speed acquisition system has already been proposed [56], showing that acquisition can be performed at a very high rate if necessary. But data are post processed, for off-line display. We have studied improvement of tomographic diffractive microscopy, by making use of Graphical Processor Unit reconstruction of the images, and shown that several 3-D images per second can be reconstructed and displayed. Coupled with ultrafast acquisition, such a system would be useful to study in real-time living specimens, or dynamic phenomena, provided that data transfer from camera to computer is high enough. For example, photopolymerisation micro- and nano-manufacturing processes could be monitored in situ, as the refraction index of resins varies when transformed from liquid to solid phase [82]. Presently, the control is performed after fabrication and rinsing of the liquid resin, to isolate the fabricated sample. In biology, cell tracking and sorting could be improved and accelerated, for example for non-fluorescence cytometry [83], which constitutes an interesting alternative for studies requiring to work on unlabeled cells. Having live 3-D views and precise measurements on oocytes and sperm cells [84,85] may also help for in-vitro fertilization studies. For example, morphological features of egg cells are up-to-now measured using 2-D only light microscopy images [84], and could benefit from the superior imaging capabilities TDM allows for.

Tomographic diffractive microscopy in reflection [39,40] could also benefit from fast GPU image reconstructions. 
Then, a possible improvement for further accelerating acquisition would be to use advanced reconstruction methods, which require much less data for precise specimen reconstructions than direct Fourier transform [86-90]. The reconstruction speed may be lower, as in particular iterative methods are slower, but acquisition could be simplified and accelerated, and such approaches could also benefit from much faster GPU reconstructions.

Published work on TDM [17-19,27,41-44,53,54,86-91] have clearly demonstrated the usefulness of accessing the index of refraction distribution at microscopic scale, for biological research as well as work on artificial samples. But note that absorption is often not discussed, while it has been shown that refraction and absorption indeed provides complementary information [25,26,32,37,38,45-47]. This dual imaging capability should be further investigated, as absorption measurements may probably also contribute to diagnostic tools. Note that low coherence setups provide simplified hardware [91], or, alternately, simplified amplitude and phase detection approaches that do not require a separate reference beam for interferometry [7,92-95]. In some cases, one can benefit from the movements of the sample itself to perform tomographic reconstructions, which could also contribute to design simpler systems [96].

To conclude, we believe that combining rapid-acquisition, simplified setups, with fast GPU reconstruction and real-time 3-D display of the observed sample images could greatly accelerate for wide adoption of tomographic imaging by non-specialists. While such a real-time $3-\mathrm{D}$ microscopy system has yet to be developed, all elementary bricks are now available, and we believe that such a real-time TDM could then constitute no less than a high-tech replacement of standard transmission microscopy, in which samples could be imaged, reconstructed and displayed in 3-D in live conditions (several 3-D images per seconds), even for large-scale images $\left(512^{3}\right.$ voxels or more).

This work was funded by French National Research Agency (ANR) under JCJC2011-OSIRIS (Optical Scanning, Isotropic Resolution Imaging System) grant. We thank the Nvidia Academic Research Program for granting a Tesla C2075 card.

\section{References}

1. G. Popescu, Quantitative Phase Imaging of Cells and Tissues, McGraw Hill, 2011.

2. Myung K. Kim, Digital Holographic Microscopy: Principles, Techniques, and Applications, Springer Series in Optical Science Vol. 162, Springer, 2011.

3. B. Kemper, Digital holographic microscopy: quantitative phase imaging and applications in live cell analysis, in Handbook of Coherent-Domain Optical Methods, V. V. Tuchin, ed., 215-257, Springer, 2013.

4. K. Lee, K. Kim, J. Jung, J. Heo, S. Cho, S. Lee, G. Chang, Y. Jo, H. Park, and Y. Park, Quantitative Phase Imaging Techniques for the Study of Cell Pathophysiology: From Principles to Applications, Sensors 13, 4170-4191 (2013).

5. R. Carriles, D. N. Schafer, K. E. Sheetz, J. J. Field, R. Cisek, V. Barzda, A. W. Sylvester, and J. A. Squier, "Invited Review Article: Imaging techniques for harmonic and multiphoton absorption fluorescence microscopy", Rev. Sci. Instrum. 80, 081101 (2009).

6. E. Cuche, P. Marquet, and C. Depeursinge "Simultaneous amplitude-contrast and quantitative phasecontrast microscopy by numerical reconstruction of Fresnel off-axis holograms" Appl. Opt. 38, 69947001 (1999).

7. P. Bon, G. Maucort, B. Wattellier, and S. Monneret, "Quadriwave lateral shearing interferometry for quantitative phase microscopy of living cells," Opt. Express 17, 13080-13094 (2009).

8. J. Kim, J.-H. Jung, Y. Jeong, K. Hong, and B. Lee "Real-time integral imaging system for light field microscopy" Opt. Exress 22, 10210-10220 (2014).

9. S. S. Kou, L. Waller, G. Barbastathis, and C.J.R. Sheppard, "Transport-of-intensity approach to differential interference contrast (TI-DIC) microscopy for quantitative phase imaging" Opt. Lett. 35, 447-449 (2010). 
10. S. V. King, A. Libertun, R. Piestun, C. J. Cogswell, and C. Preza, "Quantitative phase microscopy through differential interference imaging", J. Biomed. Opt. 13, 024020 (2008)

11. See for example: https://www.lynceetec.com http://www.phiab.se http://www.ovizio.com http://phasicscorp.com

12. M. K. Kim, "Principles and techniques of digital holographic microscopy" SPIE Review 1, 018005 (2010).

13. Biomedical Optical Phase Microscopy and Nanoscopy, Edited by N. T. Shaked, Z. Zalevsky, and L. Satterwhite, (Elsevier, 2012).

14. M. Debailleul, B. Simon, V. Georges, O. Haeberlé, and V. Lauer, "Holographic microscopy and diffractive microtomography of transparent samples," Meas. Sci. Technol. 19, 074009 (2008).

15. A. Barty, K. A. Nugent, A. Roberts, and D. Paganin, "Quantitative phase tomography," Opt. Commun. 175, 329-336 (2000).

16. M. Fauver, E. J. Seibel, J. R. Rahn, M. G. Meyer, F. W. Patten, T. Neumann, and A. C. Nelson, "Three-dimensional imaging of single isolated cell nuclei using optical projection tomography," Opt. Exp. 13, 4210-4223 (2005).

17. F. Charrière, A. Marian, F. Montfort, J. Kuehn, T. Colomb, E. Cuche, P. Marquet, and C. Depeursinge, "Cell refractive index tomography by digital holographic microscopy," Opt. Lett. 31, 178180 (2006).

18. F. Charrière, N. Pavillon, T. Colomb, T. Heger, E. Mitchell, P. Marquet, B. Rappaz, and C. Depeursinge, "Living specimen tomography by digital holographic microscopy: morphometry of testate amoeba", Opt. Express 14, 7005-7013 (2006).

19. A. Kuś, M. Dudek, B. Kemper, M. Kujawińska, and A. Vollmer, "Tomographic phase microscopy of living three-dimensional cell cultures" J. of Biomed. Opt. 19, 046009 (2014).

20. M. Habaza, B. Gilboa, Y. Roichman, and N. Shaked, "Tomographic phase microscopy with $180^{\circ}$ rotation of live cells in suspension by holographic optical tweezers", Opt. Lett. 40, 1881-1884 (2015).

21. M. Habaza, M. Kirschbaum, C. Guernth-Marschner, G. Dardikman, I. Barnea, R. Korenstein, C. Duschl, and N. T. Shaked, "Rapid 3D refractive-index imaging of live cells in suspension without labeling using dielectrophoretic cell rotation” Adv. Sci., 1600205 (2016)

22. Y.-C Lin, H.-C. Chen, H.-Y. Tu, C.-Y. Liu, and C.-J. Cheng, "Optically driven full-angle sample rotation for tomographic imaging in digital holographic microscopy" Opt. Lett. 42, 1321-1324 (2017)

23. S. Kawata, O. Nakamura, T. Noda, H. Ooki, K. Ogino, Y. Kuroiwa, and S. Minami, "Laser computedtomography microscope", Appl. Opt. 29, 3805-3809 (1990).

24. T. Noda, S. Kawata, and S. Minami, "Three-dimensional phase-contrast imaging by a computedtomography microscope", Appl. Opt. 31, 670-674 (1992).

25. V. Lauer, "New approach to optical diffraction tomography yielding a vector equation of diffraction tomography and a novel tomographic microscope," J. Microsc. 205, 165-176 (2002).

26. B. Simon, M. Debailleul, V. Georges, V. Lauer, and O. Haeberlé, "Tomographic diffractive microscopy of transparent samples," Eur. Phys. J. Appl. Phys. 44, 29-35 (2008).

27. R. Fiolka, K. Wicker, R. Heintzmann, and A. Stemmer, "Simplified approach to diffraction tomography in optical microscopy", Opt. Express 17, 12407-12417 (2009).

28. S. Chowdhury, W. J. Eldridge, A. Wax, and J. Izatt, "Refractive index tomography with structured illumination" Optica 4, 537-545 (2017)

29. O. Haeberlé, K. Belkebir, H. Giovannini and A. Sentenac, "Tomographic diffractive microscopy: basics, techniques and perspectives", J. Mod. Opt. 57, 686-699 (2010).

30. D. Jin, R. Zhou, Z. Yaqoob, and P. T. C. So, "Tomographic phase microscopy: principles and applications in bioimaging" J. Opt. Soc. Am. B 34, B64 (2017)

31. S. Vertu, J. Flügge, J.-J. Delaunay, and O. Haeberlé, "Improved and isotropic resolution in tomographic diffractive microscopy combining sample and illumination rotation," Central Eur. J. Phys. 9, 969-974 (2011).

32. B. Simon, M. Debailleul, M. Houkal, C. Ecoffet, J. Bailleul, J. Lambert, A. Spangenberg, H. Liu, O. Soppera and O. Haeberlé "Tomographic diffractive microscopy with isotropic resolution", Optica 4, 460$463(2017)$

33. E. Wolf, "Three-dimensional structure determination of semi-transparent objects from holographic data", Opt. Commun. 1, 153-156 (1969). 
34. J. Goodman, Introduction to Fourier Optics (McGraw-Hill, 2008).

35. J. D. Jackson, Classical Electrodynamics, (Wiley-New York, 1999).

36. S. Inoue, and K. R. Spring, Video Microscopy: The Fundamentals (Plenum Press-New York, 1997).

37. M. Debailleul, V. Georges, B. Simon, R. Morin, and O. Haeberlé, "High-resolution three-dimensional tomographic diffractive microscopy of transparent inorganic and biological samples," Opt. Lett. 34, 7981 (2009).

38. B. Simon, M. Debailleul, A. Beghin, Y. Tourneur, and O. Haeberlé, "High resolution tomographic diffractive microscopy of biological samples," J. Biophotonics 3, 462-467 (2010).

39. M. Sarmis, B. Simon, M. Debailleul, B. Colicchio, V. Georges, J.-J. Delaunay, and O. Haeberlé, "High resolution reflection tomographic diffractive microscopy,” J. Mod. Opt. 57, 740-745 (2010).

40. H. Liu, J. Bailleul, B. Simon, M. Debailleul, B. Colicchio, and O. Haeberlé, "Tomographic diffractive microscopy and multiview profilometry with flexible aberration correction", Appl. Opt. 53, 748-755 (2014).

41. Y. Cotte, F. Toy, P. Jourdain, N. Pavillon, D. Boss, P. Magistretti, P. Marquet and C. Depeursinge, "Marker-free phase nanoscopy", Nature Phot. 7, 113-117 (2013).

42. W. Choi, C. Fang-Yen, K. Badizadegan, S. Oh, N. Lue, R. R. Dasari, and M. S. Feld, "Tomographic phase microscopy", Nature Meth. 4, 717-719 (2007).

43. T. Kim, R. Zhou, M. Mir, S. Derin Babacan, P. Scott Carney, L. L. Goddard, and G. Popescu, "Whitelight diffraction tomography of unlabelled live cells", Nature Phot. 8, 256-263 (2014).

44. B. Bhaduri, C. Edwards, H. Pham, R. Zhou, T. H. Nguyen, L. L. Goddard, and G. Popescu, "Diffraction phase microscopy: principles and applications in materials and life sciences", Adv. Opt. Photon. 6, 57-119 (2014).

45. K. Kim, S. Lee, J. Yoon, J. Heo, C. Choi, and Y. Park, "Three-dimensional label-free imaging and quantification of lipid droplets in live hepatocytes", Scientific Reports 6, 36815 (2016)

46. T. C., Wedberg, and W. C. Wedberg, Tomographic reconstruction of the cross-sectional refractive index distribution in semi-transparent, birefringent fibres, J. Microsc. 177, 53-67 (1995).

47. K. Kim, H. Yoon, M. Diez-Silva, M. Dao, R. R. Dasari, and Y. Park, High-resolution threedimensional imaging of red blood cells parasitized by Plasmodium falciparum and in situ hemozoin crystals using optical diffraction tomography, J. Biom. Opt. 19, 011005 (2014).

48. F. Montfort, T. Colomb, F. Charrière, J. Kühn, P. Marquet, E. Cuche, S. Herminjard, and C. Depeursinge, "Submicrometer optical tomography by multiple-wavelength digital holographic microscopy", Appl. Opt. 45, 8209-8217 (2006).

49. J. Kuihn, F. Montfort, T. Colomb, B. Rappaz, C. Moratal, N. Pavillon, P. Marquet, and C. Depeursinge, "Submicrometer tomography of cells by multiple-wavelength digital holographic microscopy in reflection", Opt. Lett. 34, 653-655 (2009).

50. S. Vertu, I. Yamada, J.-J. Delaunay, and O. Haeberlé, "Tomographic observation of transparent objects under coherent illumination and reconstruction by filtered backprojection and Fourier diffraction theorem", Proc. SPIE 6861, 686103 (2008).

51. S. Vertu, J.-J. Delaunay, I. Yamada, and O. Haeberlé, "Diffraction microtomography with sample rotation: influence of a missing apple core in the recorded frequency space", Centr. Eur. J. Phys. 7, 2231 (2009).

52. Y.-C. Lin, and C.-J. Cheng, "Sectional imaging of spatially refractive index distribution using coaxial rotation digital holographic microtomography", J. Opt. 16, 065401 (2014).

53. B. Bhaduri, H. Pham, M. Mir, and G. Popescu, "Diffraction phase microscopy with white light", Opt. Lett. 37, 1094-1097 (2012).

54. Z. Wang, D. L. Marks, P. Scott Carney, L. J. Millet, M. U. Gillette, A. Mihi, P. V. Braun, Z. Shen, S. G. Prasanth, and G. Popescu, "Spatial light interference tomography (SLIT)", Opt. Express 19, 1990719918 (2011).

55. J. Bailleul, "Microscopie tomographique diffractive à résolution isotrope - Isotropic resolution tomographic diffractive microscopy", PhD Thesis, 23. September 2016, Université de Haute-Alsace

56. M. Kim, Y. Choi, C. Fang-Yen, Y. Sung, R. R. Dasari, M. S. Feld, and W. Choi, "High-speed synthetic aperture microscopy for live cell imaging, Opt. Lett. 36, 148-150 (2011).

57. K. Kim, K. Sang Kim, H. J. Park, J. Chul Ye, and Y. K. Park, "Real-time visualization of 3-D dynamic microscopic objects using optical diffraction tomography", Opt. Expr. 21, 32269-32278 (2013) 
58. G. Dardikman, M. Habaza, L. Waller, and N. T. Shaked, "Video-rate processing in tomographic phase microscopy of biological cells using CUDA", Opt. Express 24, 11839-11854 (2016)

59. A. Fog, "Optimizing Software in C++", Online book (2014), http://www,agner.org/optimize

60. G. Hager and G. Wellein, Introduction to High Performance Computing for Scientists and Engineers (CRC Press, Inc., 2010).

61. V. Lee, C. Kim, J. Chhugani, M. Deisher, D. Kim, A. Nguyen, N. Satish, M Smelyanskiy, S. Chennupaty, P. Hammarlund, R. Singhal, and P. Dubey, "Debunking the 100X GPU vs CPU Myth: An Evaluation of Throughput Computing on CPU and GPU”, SIGARCH Comput. Archit. News 38, 451460 (2010).

62. J. Bailleul, B. Simon, M. Debailleul, H. Liu, and O. Haeberlé, "GPU acceleration towards real-time image reconstruction in 3D tomographic diffractive microscopy, Proc. SPIE 8437, Paper 8437-7 (2012)

63. http://www.fftw.org

64. C. Lomont, "Introduction to Intel Advanced Vector Extensions", (Intel, 2012). https://software.intel.com/en-us/articles/introduction-to-intel-advanced-vector-extensions

65. M. Frigo and S. Johnson, "The Design and Implementation of FFTW3", Spec. Issue of Prog. Generation, Optimization and Platform Adaptation 93, 216-231 (2005).

66. O. Haeberlé, J. Bailleul, H. Liu, B. Simon, and M. Debailleul, "Image acquịsition, processing and reconstruction in holographic and tomographic diffractive microscopy," in Imaging and Applied Optics 2014, OSA Technical Digest (online) (Optical Society of America, 2014), paper DM3B.1.

67. V. V. Volkov and Y. Zhu, "Deterministic phase unwrapping in the presence of noise", Opt. Lett. 28, 2156-2158 (2003)

68. M. Herráez, D. R. Burton, M. J. Lalor, and M. A. Gdeisat, "Fast two-dimensional phase-unwrapping algorithm based on sorting by reliability following a noncontinuous path", Appl. Opt. 41, 7437-7444 (2002)

69. Intel Corporation, Intel 64 and ia-32 architectures developer's manual: Vol. 1. Technical Report 253665-057US, Intel, Inc., December 2015.

70. Intel Corporation, Intel ${ }^{\circledR}$ Xeon Phi ${ }^{\mathrm{TM}}$ Coprocessor Instruction Set Architecture Reference Manual, Reference Number: 327364-001, September 7, 2012.

71. B. Chapman, G. Jost and R. Pas, Using OpenMP: Portable Shared Memory Parallel Programming (The MIT Press, 2007).

72. R. Farber, CUDA Application Design and Development (Morgan Kaufmann, 2012).

73. O. Backoach, S. Kariv, P. Girshovitz, and N. T. Shaked, "Fast phase processing in off-axis holography by CUDA including parallel phase unwrapping", Opt. Expr. 24, 3177-3188 (2016)

74. M. Pyka, M. Hertog, R. Fernandez, S. Hauke, D. Heider, U. Dannlowski, and C. Konrad, "FMRI data visualization with brainblend and blender", Neuroinformatics 8, 21-31 (2010).

75. Y. Pan, J. Liu, X. Li, and Y. Wang, "A review of dynamic holographic three-dimensional display: Algorithms, devices, and systems", IEEE Trans. Indus. Informatics 12, 599-1610 (2016).

76. R. H.-Y. Chen and T. D. Wilkinson "Computer generated hologram with geometric occlusion using GPU-accelerated depth buffer rasterization for three-dimensional display", Appl. Optics 48, 4246-4255 (2009).

77. Y. Pan, X. Xu, S. Solanki, X. Liang, R. Tanjung, C. Tan, and T. Chong, "Fast CGH computation using s-LUT on GPU", Opt. Express 17, 18543-18555 (2009).

78. T. Shimobaba, T. Ito, N. Masuda, Y. Ichihashi, and N. Takada, "Fast calculation of computergenerated- hologram on AMD hd5000 series GPU and OpenCL”, Opt. Express 18, 9955-9960 (2010).

79. E. Bruckheimer, C. Rotschild, T. Dagan, G. Amir, A. Kaufman, S. Gelman, and E. Birk, "Computergenerated real-time digital holography: first time use in clinical medical imaging", European Heart Journal-Cardiovascular Imaging 17, 845-849 (2016).

80. E. Bruckheimer and C. Rotschild, "Holography for imaging in structural heart disease", EuroIntervention: J. of EuroPCR 12, X81-X84 (2016).

81. Y.-H. Huang, T.-C. Yu, P.-H. Tsai, Y.-X. Wang, W.-L. Yang, and M. Ouhyoung, "Scope+: A stereoscopic video see-through augmented reality microscope", in Adjunct Proceedings of the 28th Annual ACM Symposium on User Interface Software \& Technology, 33-34 (2015).

82. X. Zhou, Y. Hou, and J. Lin, "A review on the processing accuracy of two-photon polymerization", AIP Advances 5, 030701 (2015). 
83. E. Luther, L. P. Mendes, J. Pan, D. F. Costa, V. P. Torchilin "Applications of label-free, quantitative phase holographic imaging cytometry to the development of multi-specific nanoscale pharmaceutical formulations", Cytometry A 91, 412-423 (2017).

84. E. S. Filho, J. Noble, and D. Wells, "A Review on Automatic Analysis of Human Embryo Microscope Images", The Open Biomedical Engineering Journal 4, 170-177 (2010).

85. G. Di Caprio, M. A. Ferrara, L. Miccio, F. Merola, P. Memmolo, P. Ferraro, and G. Coppola, "Holographic imaging of unlabelled sperm cells for semen analysis: a review", J. Biophoton 8, 779-789 (2015).

86. Y. Sung, W. Choi, C. Fang-Yen, K. Badizadegan, R. R. Dasari, M. S. Feld, "Optical diffraction tomography for high resolution live cell imaging", Opt. Express 17, 266-277 (2009).

87. S. J. LaRoque, E. Y. Sidky, and X. Pan, "Accurate image reconstruction from few-view and limitedangle data in diffraction tomography", J. Opt. Soc. Am. A 25, 1772-1782 (2008).

88. J.-W. Su, W.-C. Hsu, C.-Y. Chou, C.-H. Chang, K.-B. Sung, "Digital holographic microtomography for high-resolution refractive index mapping of live cells", J. Biophotonics 6, 416-424 (2013).

89. Y. Sung, R. R. Dasari, "Deterministic regularization of three-dimensional optical diffraction tomography”. J. Opt. Soc. Am. A. 8, 1554-1561 (2011).

90. C. Ding, K. Yang, W. Li, W. Guo, X. Zhang, M. Xia, "Application of diffraction tomography theory to determine size and shape of spheroidal particles from light scattering", Opt. Laser Tech. 62, 135-140 (2014).

91. J. Soto, J. Rodrigo, and T. Alieva, "Label-free quantitative 3D tomographic imaging for partially coherent light microscopy", Opt. Express 25, 15699-15712 (2017).

92. B. Kemper, A. Vollmer, C. E. Rommel, J. Schnekenburger, G. von Bally, "Simplified approach for quantitative digital holographic phase contrast imaging of living cells," J. Biomed. Opt. 16, 026014 (2011).

93. P. Gao, B. Yao, J. Min, R. Guo, J. Zheng, T. Ye, I. Harder, V. Nercissian, and K. Mantel, "Parallel twostep phase-shifting point-diffraction interferometry for microscopy based on a pair of cube beamsplitters", Opt. Express 19, 1930-1935 (2011).

94. N.-I. Toto-Arellano, V. H. Flores-Muñoz, and B. Lopez-Ortiz, "Dynamic phase imaging of microscopic measurements using parallel interferograms generated from a cyclic shear interferometer", Opt. Express 22, 20185-20192 (2014).

95. Y. Ruan, P. Bon, E. Mudry, G. Maire, P. C. Chaumet, H. Giovannini, K. Belkebir, A. Talneau, B. Wattellier, S. Monneret, and A. Sentenac, "Tomographic diffractive microscopy with a wavefront sensor", Opt. Lett. 37, 1631-1633 (2012).

96. F. Merola, P. Memmolo, L. Miccio, R. Savoia, M. Mugnano, A. Fontana, G. D'Ippolito, A. Sardo, A. Iolascon, A. Gambale and P. Ferraro, "Tomographic flow cytometry by digital holography", Light: Science \& Applications 6, e16241 (2017) 